http://dx.doi.org/10.18778/2196-8403.2016.06

EDYTA BŁACHUT

\title{
Anspielungstypen. Eine empirisch-linguistische Auseinandersetzung
}

Celem artykułu jest przedstawienie sposobów formułowania wypowiedzi aluzyjnych, zaczerpniętych z wybranych niemieckich artykułów prasowych dotyczących wydarzeń politycznych i ekonomicznych. Dokonano charakterystyki językoznawczej różnych typów wypowiedzi podtekstowych, które powstały $\mathrm{m}$. in. poprzez odwołanie się do utrwalonych form językowych (np. cytatów, przysłów, metafor itp.) lub za pomocą gier słownych. Zwrócono uwagę na możliwości rozpoznania form wyjściowych przez odbiorcę, stanowiących bazę do formułowania wypowiedzi podtekstowych. W kolejnym kroku podjęto próbę interpretacji funkcji, jakie analizowane wypowiedzi aluzyjne pełnią $\mathrm{w}$ danym tekście.

Der Beitrag präsentiert eine Übersicht über verschiedene Anspielungstypen, die in der deutschen Tagespresse im politischen und wirtschaftlichen Diskurs zu verzeichnen sind. Vorgenommen werden linguistische Analysen zu belegten Anspielungstypen nach ihrer Form (Wortspiele, Abwandlungen formelhafter Wendungen, Metaphern u. a.) und der Wahrscheinlichkeit, mit der die jeweilige Anspielung als solche vom Empfänger erkannt wird. Im zweiten Schritt wird versucht, zu ermitteln und zu begründen, welche Funktionen sich den Anspielungen im konkreten Textzusammenhang zuschreiben lassen.

The article presents an overview of different types of allusive statements, using original examples from German press articles in the field of politics and economics. In the first part, a linguistic analysis of the form of allusive statements, which is often based on word-play, modifications of idioms, metaphors, etc., has been carried out. In the second part, the article demonstrates and explains the functions which can be ascribed to the allusive statements, with references to context, situation and intention of the language user. 


\section{Zur Einleitung: Definitorisches}

Der Duden. Deutsches Universalwörterbuch (2015) verzeichnet im Lemma des Verbs anspielen u. a. die Bedeutung „versteckt hinweisen“. Wenn diese Definition auf das linguistische Untersuchungsfeld übertragen wird, können unter der Bezeichnung Anspielung zum Beispiel Formulierungen wie Bleibe im Lande... und nähre dich deftig (taz 07.10.1989, S. 26) oder Visa frei bis Hawaii (taz 06.11.1989, S. 2) verstanden werden. Bezugspunkt für beide Formulierungen ist ein konkretes geschichtliches Ereignis. Nur bei entsprechendem Weltwissen erkennen die Empfänger eine Anspielung als Anspielung (als Prozess) und sind in der Lage festzustellen, dass ihnen etwas ,versteckt ${ }^{\star}$ vermittelt werden soll. Damit ist wiederum in sprachlicher Hinsicht verbunden, dass Ausdrücke (als Effekt des Anspielens), je indirekter formuliert und je ästhetisch ansprechender sie in ihrer Form sind, desto stärker von sprachlicher Kreativität zeugen. Anspielungstypen lassen sich nicht auf eine Form begrenzen.

Mit Anspielungen beschäftigen sich verschiedene Wissenschaftszweige, z. B. die Soziolinguistik und Psycholinguistik in der Sprachwissenschaft oder die Rhetorik und Stilistik in der Literaturwissenschaft. Im Blickfeld moderner linguistischer Untersuchungen bleiben nach wie vor die Sprechakttheorie und ein Teilbereich der Phraseologie als jene Disziplinen, die dem Begriff wissenschaftlich Kontur gegeben haben und den sich andere Disziplinen (z. B. die Diskurs- oder Gesprächsforschung, die die Sprechkonventionen von Gruppen, Sprachgemeinschaften und Individuen unter soziologischem Aspekt untersuchen) zu eigen machen. Interessant ist hierbei die Frage, wie Sprechakttheorie und Phraseologie die Anspielung erklären. Dies soll anhand der bereits eingangs zitierten Formulierungen Bleibe im Lande... und nähre dich deftig (taz 07.10.1989, S. 26) und Visa frei bis Hawaii (taz 06.11.1989, S. 2) veranschaulicht werden.

Mit Anspielungen als Sprechakt hat sich z. B. RÖMER (1977) beschäftigt. Sie unterscheidet dabei zwischen Anspielungen und Andeutungen (als indirekte Sprechakte), indem sie Andeutungen die Vermittlung neuer Informationen zuschreibt, Anspielungen dagegen nicht. Zum erwähnten Abgrenzungskriterium von RÖMER lässt sich folgendes formulieren: Man kann wohl annehmen, dass jede Art, einen Hinweis, aus welchen Gründen auch immer, ,versteckt‘ zu vermitteln, zugleich etwas Neues ins Gespräch einführen kann oder auch etwas Neues für den Kommunikationspartner darstellt. Wenn man sich die oben angeführten Überschriften noch einmal anschaut, so kann man feststellen, dass 
sie beide Formulierungen sind, die auf Ereignisse und Verhältnisse um die Wendezeit 1989/1990 anspielen. Sie suggerieren eine Forderung und sind zu einem gewissen Grad emotional aufgeladen. Gefühlswörter wie Hoffnung oder Angst u. a. werden hier zwar nicht verwendet, aber Emotionen werden indirekt, ,versteckt ${ }^{\star}$ vermittelt. Im alltäglichen Sprachgebrauch wird vermutlich zwischen Anspielung und Andeutung nicht unterschieden. An dieser Stelle sei noch einmal die Erläuterung aus dem Duden. Deutsches Universalwörterbuch (2015) zitiert, worin das eine Wort mit dem anderen erklärt wird. Eine Anspielung ist danach „Andeutung, versteckter Hinweis“.

Die angeführten Formulierungen unterscheiden sich formal. Wenn man schon der Abgrenzung bei den Begriffen Anspielung und Andeutung bedarf, könnte man in formaler Hinsicht (beim Unterscheidungskriterium neu) als Anspielung Formulierungen betrachten, denen bekannte und belegbare Ausdrücke zugrunde liegen. Bei Bleibe im Lande... und nähre dich deftig (taz 07.10.1989, S. 26) ist dies die Anspielung auf das Sprichwort Bleibe im Lande und nähre dich redlich (aus: Psalm 37, Vers 3). Als Andeutung könnten dann Ausdrücke ohne eine solche bekannte und belegbare Ausgangsbasis wie Visa frei bis Hawaii (taz 06.11.1989, S. 2) verstanden werden. Die Phraseologieforschung bietet hier eine klare Definition der Anspielung an, nämlich die einer Abwandlung (auch: Modifikation) der lexikalischen und / oder morphosyntaktischen Struktur einer usuellen, kodifizierten Wendung (eines Phraseologismus, Sprichworts, literarischen Zitats, Buch-, Film-, Liedtitels, von Aussprüchen bekannter Personen, Losungen etc.), unter der Annahme, dass die neu entstandenen Wendungen nur für die Zwecke eines Textes hergestellt wurden, also okkasionell bleiben. Des Terminus Anspielung in diesem Sinne bedienen sich u. a. WiLSS (1980, 1989) und SAUER (1998); bei SAUER sind Anspielungen eine Untergruppe der kontextuellen (Sprach-) Spiele.

Im Folgenden werden unter dem Terminus Anspielungstypen alle sprachlich auffälligen Formen des Anspielens besprochen.

\section{Zum Untersuchungskorpus}

Bezugspunkt für Anspielungen im öffentlichen Raum kann jedes historische oder zeitgenössische Ereignis, jede bekannte Person, ihr Aussehen oder ihre Verhaltensweise, Handlungen, Entscheidungen, Überzeugungen etc. sein. Ich betrachte Anspielungen im Sinne von WILSS (1989:VII) als Teile kollektiver 
und diskursiver Welterfahrung und Weltwahrnehmung. Die im Beitrag diskutierten Anspielungen sind der deutschen überregionalen Tagespresse (taz und F.A.Z.) in politischen und wirtschaftlichen Diskussionsbereichen um 1989 und 2007 entnommen. Besonders das Jahr 1989 suggeriert die eindeutige Anbindung an den historischen Kontext. Dem entgegen ist aber die Analyse nicht diskursbezogen gemeint. Ich verwende für die Zwecke dieses Beitrags ein selbst erstelltes Belegkorpus, um verschiedene, sprachlich interessante Anspielungsformen herauszufiltern und sie nach verwendeten lexikalischen, grammatischen, stilistischen, kompositionellen u. a. Anspielungstechniken zu klassifizieren. Im zweiten Schritt werde ich versuchen zu erkennen und zu begründen, welche Funktionen sich diesen Anspielungen zuschreiben lassen. Das Belegkorpus zählt über tausend Einzelexemplare, es wird jedoch keine statistische Auswertung angeboten. Für die Form, die Anspielungen in diesem konkreten Belegkorpus annehmen, werden prototypische Beispiele angeführt.

\section{Anspielungstypen}

Die gesammelten Belege dokumentieren, dass für Anspielungen vielfältige sprachliche Möglichkeiten zur Verfügung stehen. Im Folgenden werden auffällige Anspielungstypen in den Fokus der empirischen Auseinandersetzung gestellt. Diskutiert werden die Bildungsweise und der Mechanismus für die Aufdeckung der Anspielung.

\subsection{Veränderungen der äußeren Form einer Wendung}

Unter Wendung werden alle Arten von festen Wortverbindungen im engeren und weiteren Sinne gefasst, d. h. stereotype und idiomatische Ausdrücke wie Phraseologismen, Sprichwörter und Floskeln bis hin zu Zitaten, darunter Schlagzeilen, Titel, Losungen etc. Es sind Ausdrücke, die samt ihrer Form und ihrer Bedeutung bzw. dem spezifischen Verwendungskontext im Sprachbewusstsein der Sprecher verankert sind. Der Bekanntheitsgrad solcher Ausdrücke oder wenigstens die Annahme darüber mag ein hauptsächlicher Grund dafür sein, dass sie zum häufigen Sprachmittel der Anspielung auf außersprachliche Erscheinungen im öffentlichen Raum werden. Die beabsichtigte Veränderung (Modifizierung) der Ausdrücke ist einerseits ein Zeichen der Kreativität der Autoren, sie verlangt andererseits aber ebenso Kreativität vom Empfänger, und zwar im Erkennen und Verstehen des nun nicht erwartungsgemäß gebauten Textes. Diese Veränderung bzw. Modifizierung des Ausdrucks kann nun (ganz im Sinne von WILSS 1989) auch als Anspielung auf den 
Ausdruck selbst betrachtet werden. Ich werde mich nun den sprachlichen Anspielungstypen in diesem Bereich zuwenden (das, worauf damit in der außersprachlichen Wirklichkeit angespielt wird, spielt für die linguistische Analyse eine kleinere Rolle). In den untersuchten Zeitungen sind Substitution, Hinzufügung und Reduktion am häufigsten belegbar. ${ }^{1}$

Die Substitution ist ein Anspielungstyp, bei dem die Struktur der Basiswendung unverändert bleibt. Dem Austausch unterliegen Laute, Morpheme, einzelne Lexeme, mehrere Lexeme und Teilsätze (im Falle von komplexen Wendungen). Einerseits sind die bewahrte Struktur, andererseits - u. a. bei der lexikalischen Substitution - die Ersetzung durch Wörter derselben Bedeutungsdomäne Kriterien, durch die die Rezipienten in der Lage sind, die Neubildung mit der Basiswendung zu assoziieren. Die lexikalische Substitution kann manchmal so weit gehen, dass das semantische Kriterium nicht erhalten bleibt. In jedem Fall dienen die Basiswendungen als Schablone mit einer oder mehreren beliebig ausfüllbaren Positionen. In den angeführten Belegen sind die ausgetauschten Teile jeweils hervorgehoben.

Beispiel 1:

Liberté, Autorité, Absurdité (taz 16./17.05.2007, S. 20)

[bezogen auf das politische Verhalten von Sarkozy]

Basiswendung: Liberté, Egalité, Fraternité

Beispiel 2:

„Freiheit, Reichheit, Käuflichkeit“ (taz 11.12.1989, S. 17)

[Anti-Wiedervereinigungsparolen]

Basiswendung: Freiheit, Gleichheit, Brüderlichkeit

Beispiel 3:

„Spygate" dicht (taz 08.03.2007, S. 12)

Die Wende wird zum ,Wandlitzgate“" (taz 04.12.1989, S. 3)

Basiswendung: watergate

Beispiel 4:

Aller guten Abschiede sind drei. (F.A.Z. 09.05.2007, S. 24)

[im Kontext der Entscheidung, das Bundestagsmandat auf Raten niederzulegen] Basiswendung: Aller guten Dinge sind drei

1 Die Modifizierungsarten des Bereichs fester Wendungen sind in der Forschung recht erfolgreich untersucht und erklärt worden, vgl. z. B. WILSS (1989), FLEISCHER (1997), Burger (1998), SAuer (1998), BŁachut (2004), PtashnyK (2005), PTASHNYK (2009), VATER (2010). Das Ziel dieses Aufsatzes ist nicht die Typologie der Modifizierungsarten. 
Beispiel 5:

Öko-Nationalisten aller Deutschländer, vereinigt euch? (taz 31.10.1989, S. 15)

Schlu $\beta$ mit dem SED - Schlaraffenland!, Betrogene aller Bezirke vereinigt euch! (taz 04.12.1989, S. 2)

Proletarier aller Länder - verjubelt euch! (taz 08.12.1989, S. 21)

Basiswendung: Proletarier aller Länder, vereinigt euch!

Die durch die Substitution veränderte Wendung wird häufig syntaktisch in den Satz eingebaut, z. B.:

Beispiel 6:

Die Leute (in Deutschland) werden endlich Abschied nehmen von der Illusion,

Deutschland gehöre den Deutschen. (F.A.Z. 04.05.2007, S. 11)

Basiswendung: Amerika den Amerikanern

Die Hinzufügung ist ein Anspielungstyp, bei dem sowohl die Struktur der Wendung als auch der Lexembestand erhalten bleiben. Grundsätzlich werden nominale Bestandteile durch Attribute erweitert, seltener werden sie syntaktisch, z. B. durch einen Relativsatz, abgetrennt. Auch die ganze, innerlich nicht veränderte Wendung kann erweitert werden. Den hinzugefügten Elementen kommt eine intensivierende, kommentierende oder kontrastierende Funktion zu. Die hinzugefügten Teile sind in den Beispielen hervorgehoben:

Beispiel 7:

Vom Stasi-Regen in die BND-Traufe (taz 26.10.1989, S. 1)

Basiswendung: vom Regen in die Traufe kommen ${ }^{2}$

Beispiel 8:

Das Land der unbegrenzten Online-Möglichkeiten (F.A.Z. 12.05.2007, S. 39)

Basiswendung: Das Land der unbegrenzten Möglichkeiten

Beispiel 9:

Lügen haben kurze Beine - Egon zeig, wie lang sind deine. (taz 06.11.1989, S. 2)

Basiswendung: Lügen haben kurze Beine

Hinzu kommen Fälle, in denen die erweiterte Wendung syntaktisch in den Satz eingebaut wird, z. B.:

Beispiel 10:

Meint er das Amerika Thomas Jeffersons, John. F. Kennedys oder Ronald Reagans, in dem nur ein toter Indianer, ein toter Gewerkschafter oder toter

2 Bei Phraseologismen mit offener Struktur wie in diesem Beispiel werden die Verben häufig weggelassen. 
Kommunist ein guter Indianer, Gewerkschafter oder Kommunist ist? (taz 22.05.2007, S. 17)

Basiswendung: Nur ein toter Indianer ist ein guter Indianer

Mit der Anspielung und der Einbindung in den Satzkontext gehen zugleich auch tiefere Veränderungen der Wendung einher, bei denen manchmal schwer feststellbar ist, ob sie beabsichtigt wurden. In folgenden Belegen wird jeweils das erste Nominallexem (Hand) erweitert, das zweite (Rock) erweitert oder ausgetauscht:

Beispiel 11:

Und so könnte es zu der schönen Pointe kommen, daß gerade diejenigen, die immer am lautesten die Wiedervereinigung forderten, lieber in einem geteilten Deutschland herrschen, als in einem vereinigten in die Rolle der ewigen Opposition abzusteigen - im Zweifelsfalle ist das machtpolitische Hemd eben näher als die gesamtdeutsche Hose. (taz 31.10.1989, S. 13)

„Dem Kohl ist das deutsch-deutsche Hemd eben näher als das europäische“, maulte auch ein Brüsseler Berufseuropäer. (taz 09.12.1989, S. 1)

Basiswendung: jmdm. ist das Hemd näher als der Rock

Die Reduktion ist ein Anspielungstyp, bei dem Laute, Morpheme, Lexeme oder Satzteile ausgelassen werden. Vor allem dort, wo Auslassungspunkte den reduzierten Teil suggerieren, wird ein breiterer Interpretationsraum eröffnet. Das schlüssige Steuerungsmittel für das Erkennen der Anspielung, für die Verknüpfung des Sachverhalts, auf den mit der reduzierten Wendung angespielt wird, ist immer der Textinhalt. Die Teile der reduzierten Wendung sind im folgenden Beispiel hervorgehoben:

Beispiel 12:

Wer einmal lügt ... (taz 07.11.1989, S. 6)

Basiswendung: Wer einmal lügt, dem glaubt man nicht, und wenn er auch die Wahrheit spricht.

\subsection{Anspielung durch die unveränderte Wendung}

Die festen Wendungen (vgl. dazu w. o.) sind auch in unveränderter Form Mittel zur Anspielung auf außersprachliche Ereignisse. Sie stehen mit ihrer festen Bedeutung als Schablone dafür, anderen eigene Urteile oder auch Vorurteile mitzuteilen. Sie werden so in die Texte eingebaut, dass der Textinhalt die Bedeutung der jeweiligen Wendung entwickelt und umgekehrt, die Wendung selbst den beschriebenen Sachverhalt kommentiert. Wendungen mit geschlossener syntaktischer Struktur werden unverändert in den Text integriert, solche mit offener Struktur werden häufig um Verbalteile oder Artikelwörter reduziert 
(u. a. in Überschriften) und syntaktisch eingebaut. In Fließtexten werden die syntaktischen Leerstellen entsprechend lexikalisch und inhaltlich ausgefüllt, Bestandteile können sich verschieben etc. Die festen Wendungen sind in den angeführten Beispielen hervorgehoben (zu ihrer textstrukturierenden Funktion s. weiter unten):

Beispiel 13:

Wolf im Schafspelz [als Überschrift]. Es bleibt eine Verfassung, bloß mit einem anderen Namen. Es ist der Wolf im Schafspelz ... [im Fließtext] (F.A.Z. 18.06.2007, S. 2)

Beispiel 14:

Steter Tropfen höhlt den Stein [als Überschrift] [...] Aus welchem Grund erklingen derlei Vorschläge jetzt auch bei euch als „Zukunftsmusik“? Steter Tropfen höhlt den Stein? [Schlusssatz] (taz 11.04.2007, S. 12)

Beispiel 15:

Unser Protest wurde systematisch als „Wasser auf die Mühlen der westdeutschen Revisionisten “ qualifiziert [...]. (taz 10.11.1989, S. 8)

\subsection{Anspielung durch Metapher. Verschiedene Metapherntypen}

Metaphern eignen sich besonders gut für Anspielungen auf Ereignisse unserer Umgebung, und zwar aus folgendem Grund: Der Sprecher wählt absichtlich einen Ausdruck (eine Metapher), um nicht direkt zu verstehen zu geben, was er gemeint hat. Der Mechanismus, der die Verwendung der Metapher mitsteuert, lässt sich wie folgt erklären: Ein eigentlich gemeinter Ausdruck wird aufgrund einer inhaltlichen Ähnlichkeitsbeziehung durch einen anderen (metaphorischen) ersetzt, wobei diese Ähnlichkeitsbeziehung darin besteht, dass der ersetzende (metaphorische) Ausdruck ähnliche Gegenstände oder Merkmale bezeichnet wie der ersetzte (tatsächlich gemeinte) Ausdruck. ${ }^{3}$ Jede Metapher operiert mit einem Bild. Dadurch wird eine bildliche Schau der politischen oder wirtschaftlichen Situation knapp, jedoch zugleich informativ geboten. Das Erkennen einer metaphorischen Anspielung hängt von der Auffassungsgabe des jeweiligen Adressaten ab.

3 Dieser Auffassung von Metapher als Übertragung eines Wortes in einen anderen, fremden Gegenstandsbereich liegt die aristotelische Bestimmung der Metapher als Übertragung zugrunde. Zu verschiedenen, in der Forschung diskutierten Theorien der Metaphern (z. B. Substitutionstheorie, Anomalietheorie, Interaktionstheorie) gibt FRIELING (1996) einen Überblick. 
Den Autoren stehen folgende Anspielungsarten zur Verfügung: Es werden Bilder (Motive) aus festen Wendungen, hauptsächlich Sprichwörtern und Phraseologismen, als Metapher verwendet. Dazu eignen sich unter anderem die nominalen Bestandteile als Hauptträger der Bedeutung. Man kann sie als konventionalisierte Metaphern bezeichnen, weil sie ähnlich wie Sprichwörter und Phraseologismen ein universal-sprachliches Phänomen sind. Dies bedeutet auch, dass der Rezipient durch konventionelle Metaphern perspektivisch immer in eine bestimmte Richtung gelenkt wird. Die Metaphern sind jeweils hervorgehoben:

Beispiel 16:

Katz-und-Maus-Spiel, [...] Tatsächlich haben sie [die Polizisten, E. B.] den ganzen Tag an einem verschwenderischen Katz-und-Maus-Spiel teilgenommen, das ihnen Gewohnheitsdemonstranten unter Vorspiegelung politischer Motive aufzwingen (F.A.Z. 08.06.2007, S. 1)

[Kommentar zum polizeilichen Einsatz während des G-8-Gipfels]

Basiswendung: mit jmdm. Katz und Maus spielen

Beispiel 17:

[...] Uns, der demokratischen Linken, böte sich die einmalige historische Chance, endlich in einem von unten geeinigten Deutschland den Frieden dauerhaft zu nutzen. Wir hätten allerdings von Neuvereinigung zu reden [...]. Der von oben gepropfte [sic!] Bismarckstaat mit seinen wahnsinnigen Folgen kann doch nicht ernsthaft und immer der Grund sein, sich des Themas zu enthalten oder gar gegen eine neue deutsche Einheit zu sein. Hartung und andere befinden sich zumindest mit einem Bein in dem Boot, welches unter Kohl und Co.s [sic!] Steuerung dahindümpelt - der will die Einheit nämlich dann nicht, wenn dieses Deutschland neutral und entmilitarisiert wäre [...]. (taz 01.12.1989, S. 19)

Basiswendungen: in einem Boot sitzen und mit einem Bein in etwas stehen; Metapher zum Konzept Boot

Konventionell (usuell, lexikalisiert) sind auch Metaphern, die aus mythologischen, biblischen, literarischen u. a. Topoi schöpfen. Die starke Konventionalisiertheit mancher Metaphern begründet, dass die Indirektheit überhaupt nicht (mehr) bewusst reflektiert werden kann. Dies sei an folgenden Beispielen aufgezeigt:

Beispiel 18:

[Die europäische Integration ...] ist ein trojanisches Pferd. (F.A.Z. 07.05.2007, S. 1); 
Trojanisches Pferd [als Überschrift-Kommentar zur Teilnahme des Chefs der „Islamischen Gemeinschaft“ an der deutschen Islamkonferenz] (F.A.Z. 09.05.2007, S. 43);

Sollen sich die Arbeitnehmer an den Unternehmen beteiligen, damit aus Arbeitern Kapitalisten werden? Ist die Mitarbeiterbeteiligung ein Trojanisches Pferd, um mehr Lohnflexibilität in die Betriebe zu tragen? (F.A.Z. 27.06.2007, S. 1);

Der Ring Christlich-Demokratischer Studenten (RCDS) wertete den Hochschulverband als ,,das trojanische Pferd [sic!] der Linkspartei PDS “ in der Nachfolge des 1970 aufgelösten Sozialistischen Deutschen Studentenbundes (SDS). (taz 07.05.2007, S. 6)

Beispiel 19:

Ein Pyrrhus-Sieg [Überschrift] Aus heutiger Sicht erweist sich der Sechstagekrieg freilich als das klassische Beispiel eines Pyrrhus-Sieges, eines Sieges, der mehr Schwierigkeiten schafft, als er Beute oder Nutzen einbringt. Die Wiedervereinigung Jerusalems ist nicht wirklich geglückt [...] (F.A.Z. 06.06.2007, S. 1);

Ein Pyrrhussieg [sic!] in Gaza? Hamas auf Hilfe von außen angewiesen. (F.A.Z. 19.06.2007, S. 8)

Beispiel 20:

Die Pandora-Akte der serbischen Politik [Überschrift zum Prozess gegen die Mörder Zoran Djindjićs] (F.A.Z. 24.05.2007, S. 3);

[...] Damit würde die „Büchse der Pandora“ geöffnet, wie das sogenannte ,,institutionelle Paket" des bisherigen Vertragsentwurfs auch genannt wird. (F.A.Z. 16.06.2007, S. 6)

Beispiel 21:

Es gibt eine Mehrheit links der Mitte im Deutschen Bundestag. Und diese Mehrheit könnte Kanzlerin Merkel stürzen und den Sozialdemokraten Beck zum neuen Kanzler machen. Erst säuselte der rote Oskar dies leise, jetzt schlägt er die Trommel und macht die SPD damit noch ganz kirre. Denn bei den Sozialdemokraten herrschen labile Verhältnisse [...]. (F.A.Z. 26.06.2007, S. 2)

Metaphern stehen (ähnlich wie feste Wendungen) entweder in den Überschriften oder sie werden in die Texte eingebaut, in jedem Fall aber ist es der Textinhalt, der die Bedeutung der Metapher entfaltet. In diesem Sinne basiert eine Anspielung auf der Annahme, dass der Leser im Stande ist, beide Bereiche, den realen, wortwörtlichen und den metaphorischen, bildlichen, miteinander zu verknüpfen. 
Eine metaphorische Anspielung verwendet auch Bilder und Motive aus Alltagsleben und Welterfahrungen, nicht nur aus Topoi, z. B. Erntezeit in der Online-Werbung (F.A.Z. 01.06.2007, S. 13), ${ }^{4}$ Erdbeben (F.A.Z. 02.07.2007, S. 10). ${ }^{5}$ In Metaphern dieser Art wird eine besonders einfache Beziehung hergestellt, die den Rezipienten oft undurchschaubare (z. B. politische oder ökonomische) Ereignisse auf die Ebene der von ihm selbst erfahrenen zwischenmenschlichen Beziehungen, Konfrontationen und Assoziationen versetzen lässt, häufig intuitiv. Im Sprachgebrauch zeichnen sich gewisse Tendenzen ab: Während eine Metapher bald verschwindet, wird die andere viel zitiert, modifiziert, erweitert und erfolgreich verbreitet, so dass sich metaphorische Reihen (mit einem metaphorischen Kern) bilden können. Besonders gut lässt sich die Erscheinung anhand thematisch zusammenhängender Texte beobachten. Im Folgenden wird eine metaphorische Reihe zum Konzept Musik für die Metaphorisierung der deutschen Wiedervereinigung und der Rolle der Politiker in diesem Prozess dargestellt (chronologisch zitiert):

Beispiel 22:

Im Mittelpunkt der Welt steht, dies haben wir, und allen voran die Medien unzweifelhaft deutlich gemacht, Deutschland, und wenn nicht einem vereinten, dann dem starken, übermächtigen, freien Teil: der Bundesrepublik. Alle anderen Konflikte der letzten Jahre auf diesem Erdball waren - berichterstattungsweise - nicht

Es ist die Überschrift. Ebenso wie bei literarischen und formelhaften Metaphern wird auch hier der Sinn der Metapher über den Textinhalt entwickelt, und die Metapher selbst wird im Fließtext wiederaufgenommen, z. B. Für die Online-Werber beginnt jetzt die Zeit der Ernte. [Begründung:] In den vergangenen zehn Jahren mussten sie eine tiefe Krise durchstehen und dabei bis ins Detail beweisen, dass die Werbung im Netz funktioniert. Das Durchhalten zahlt sich jetzt aus. (F.A.Z. 01.06.2007, S. 13)

Die Metapher steht auch als Überschrift. Das Erdbeben-Bild wird auf den politischen Bereich übertragen: [Entfaltung:] Stillstand gibt es in der Politik allein in der Rückschau; beim Blick nach vorne ist gut beraten, wer mit allem rechnet. Die deutsche Parteienlandschaft ist binnen weniger Jahre so sehr in Bewegung geraten, dass tektonische Verschiebungen anzunehmen sind. Seit die CDU 2003 ihren Stammplatz verlassen und auf FDP-Gebiet geschlittert ist, während zugleich die SPD von links in die Mitte gerückt ist, haben beide Volksparteien so gewaltig an Zustimmung verloren, dass sich links eine Partei einrichten konnte, die genauso stark ist, wie es die FDP oder die Grünen nach Jahrzehnten geworden sind [...]. (F.A.Z. 02.07.2007, S. 10) 
mehr als ein Intermezzo, oder besser: ein Vorspiel, featering [sic!] BRD gewissermaßen. Wir haben es wieder geschafft. Gold für Deutschland. Wir sind wer. (taz 06.10.1989, S. 8)

Beispiel 23:

[Es ist] der Bundesrepublik Deutschland gelungen, die Verhältnisse in der DDR zum Tanzen zu bringen. Von nun an wird der BRD-Imperialismus dort dauerhaft den Taktstock schwingen wollen - so wie er das in den famosen „Reform“Ländern Ungarn und Polen ja bereits praktiziert. (taz 26.10.1989, S. 14)

Beispiel 24:

Neue deutsche Einheit heißt nicht: Anschluß der DDR an die Bundesrepublik oder Fusion. Sie ist nicht Monotonie, sondern ein Konzert mit vielen Tönen - aus Sachsen, Mecklenburg, Thüringen und Nordrhein-Westfalen [...]. (taz 29.11.1989, S. 3)

Beispiel 25:

[...] Walter Momper muß sich nun weiterhin mit der zweiten Geige im deutschdeutschen Konzert abfinden. Denn das ist sicher: Er besitzt genug politische Eitelkeit, um mit seinem Vorschlag auch Eigeninteressen zu verbinden. Mit seiner Initiative wollte er auch dem zögernden und zaudernden Bundeskanzler wieder einmal zeigen, wie man Politik macht. (taz, 06.12.1989, S. 8)

Einen weiteren Anspielungstyp durch Metaphern bieten die Vergleichsmetaphern $^{6}$, die einen Platz im Vergleichsschema $A$ ist wie $B$ und $A$ ist $B$ einnehmen. Wie bei allen Metaphern handelt es sich bei den metaphorischen Vergleichen um Ausdrucksvarianten und Anspielungsverfahren, mit denen ein Thema an ein anderes herangetragen wird: Etwas, was schwer fass- und beschreibbar ist, wird durch einfacheres Mental-Bildhaftes ersetzt. Wie sich aus den dokumentierten Beispielen schließen lässt, kann es zur Herstellung von Vergleichen kommen, für die es bereits metaphorische Manifestationen in der Sprache gibt (sie sind usuelle Metaphern, ihre Bilder schöpfen sie z. B. aus Topoi) oder zur Herstellung von Vergleichen, die (spontan, textangemessen, ad hoc) völlig neue Konzeptverbindungen aktivieren (es entstehen kreative oder innovative Metaphern, die ihre Bilder aus dem Alltag oder dem individuellem Weltwissen des Autors schöpfen). Es bieten sich Vergleiche mit explizitem Vergleichselement an; sie werden meistens für die Thematisierung von Sachverhalten verwendet. Prädikativstrukturen werden häufiger für den metaphorischen Vergleich von Personen oder Sachverhalten verwendet, sie haben statischen Charakter. 
wie-Vergleiche, lexikalisiert, usuell:

Beispiel 26:

Wie Mücken und Elefanten [Überschrift] [...] Schon jetzt ist absehbar, was die Alternative zu einer multilateralen Lösung ist: bilaterale Handelsabkommen zwischen Industrie- und Entwicklungsländern, bei denen Letztere so viele Mitspracherechte haben wie bei einem Vertrag zwischen Mücke und Elefant. (taz 23./24.06.2007, S. 10)

Beispiel 27:

Das Streben nach der deutschen Einheit und die europäische Integration stünden nicht im Widerspruch, sondern seien vielmehr, wie die zwei Seiten einer Medaille". (taz 24.11.1989, S. 6)

wie-Vergleiche, nicht lexikalisiert, kreativ, innovativ:

Beispiel 28:

Orchester bewegen sich langsam wie tektonische Platten [...]; Ich laufe nur über das Feld, werfe ein wenig Saat aus und schaue, ob etwas dabei herauskommt. Es ist ganz wie bei altmodischem Ackerbau. (F.A.Z. 29.06.2007, S. 44)

Beispiel 29:

Was gestern noch festgefügt schien, ist heute überholt. Die Legitimation für die waffenstarrenden Blöcke in Ost und West schmilzt wie Schnee in der Sonne. (taz 13.11.1989, S. 21)

Beispiel 30:

Man wird zum Kreml-Astrologen, weil die Herrschenden fern sind wie die Gestirne. (taz 19.10.1989, S. 2)

[bezogen auf den Führungswechsel an der SED-Spitze]

Beispiel 31:

[...] der Fall der Mauer - ist so herrlich wie immer nur Silvester nach 28 dunklen Jahren sein kann. (taz 13.11.1989, S. 8)

Prädikativstrukturen, usuell / nicht usuell:

Beispiel 32:

YouTube ist heute unser Warhol (F.A.Z. 05.07.2007, S. 35)

Beispiel 33:

Medien sind das Untier im Dschungel (F.A.Z. 13.06.2007, S. 40)

Beispiel 34:

Die Tasche einer Frau ist eine Matrioschka (taz 15.06.2007, S. 20) 
Beispiel 35:

Die sportliche und olympische Gemeinschaft, die stark ist durch ihre Werte, welche den Sockel des Raumes von Frieden, Freiheit und Brüderlichkeit bilden, und durch den hervorragenden Platz, den sie in unserer Gesellschaft einnimmt, [kann] zum Herold der weltweiten Mobilisierung werden. (F.A.Z. 09.06.2007, S. 10)

Beispiel 36:

[X ist] Robin Hood der HVB; Robin Hood der Streubesitzeigner (F.A.Z. 29.06.2007, S. 20)

Abschließend wird noch ein interessantes, in seiner Form komplexes Anspielungsverfahren durch Metaphern vorgestellt, dessen Effekt der sogenannte Metaphorisierungstext ${ }^{7}$ ist. Der Begriff bezieht sich auf einen geschlossenen Text bzw. den Ausschnitt eines Textes, in dem metaphorische Ausdrücke mit den nicht metaphorischen zu einem harmonischen sprachlichen Gesamtkontext geflochten werden. Gut formulierte Metaphorisierungstexte sind so durchsichtig konstruiert, dass der assoziative Raum um die metaphorischen Ausdrücke Schritt für Schritt entfaltet wird. Im nachfolgenden Textauszug ist „die Uneinigkeit der europäischen Wertegemeinschaft" der metaphorisierte Sachverhalt, der seine Konzeptualisierung als Schiff findet:

Beispiel 37:

Mit preußischem Glanz und Gloria ging die Lotsin nicht von Bord, da waren die Polen vor. [Metaphorisierung von Merkel durch eine Berufsbezeichnung aus dem Seewortschatz] Doch kann Angela Merkel mit sich und ihrem Werk zufrieden sein. Ihren Auftrag hat die scheidende Ratspräsidentin erfüllt. Das Schiff namens EU, das, mit dem Gewicht des Verfassungsvertrages beschwert, auf Grund gelaufen war, liegt zwar immer noch tief im Wasser. Es schwimmt aber wieder. [Metaphorisierung der aktuellen Situation der EU] Dafür musste manches über Bord geworfen werden, dem einige nachtrauern, andere jedoch nicht [dadurch wird die Zerstrittenheit der EU-Mitglieder im Grundsätzlichen metaphorisiert, sowie die Tatsache, wie unnachgiebig sie bei der Verfolgung eigener Interessen sind]. Falls noch jemand Illusionen über die Natur dieser disparaten Reisegesellschaft gehabt haben sollte [Metaphorisierung der EU-Politiker / Länder] - am Wochenende wurden sie von Deck gefegt. [metaphorisches Bild der Schlussentscheidung] Die Uneinigkeit der europäischen ,Wertegemeinschaft" [Metaphorisierungsobjekt] zeigte sich schon in der Aufgabenstellung für die deutsche Ratspräsident-

Zum Begriff vgl. PoHL (2002:105), zur Analyse von Metaphorisierungstexten vgl. BŁACHUt (2008:44f.), BŁACHUT (2014:394f.). 
schaft, die einer „mission impossible“ [Einschätzen der Möglichkeiten einer Aufgabe durch Anspielen auf einen Filmtitel] gleichkam. [...] Die Zwillinge Kaczynski, die nach Hollywood-Manier als der gute und der böse Pole auftraten [Metaphorisierung von Verhalten durch den Vergleich aus dem Bereich des Konzepts Kino], stehen geradezu idealtypisch für eine Haltung, die der EU noch schwer zu schaffen machen wird: nehmen, was man bekommen kann, und möglichst nichts dafür geben. [...] So konnte die Bundeskanzlerin, die an die Grenzen ihrer diplomatischen Möglichkeiten ging, den Zwillingen zwar die Wurzel ziehen, den Zahn aber nicht [Zusammenfassung durch eine Metapher aus einem anderen Konzeptbereich; ein Zusammenbringen der aktuellen Situation mit der Situation, die konventionell bewertet wird, d. i. Zahnarztbesuch]. (F.A.Z. 25.06.2007, S. 1)

\subsection{Anspielung durch Wortspiele}

Wortspiele sind ein besonders anschaulicher Anspielungstyp, der häufig mit Anspielungen durch Mehrdeutigkeiten im lexikalisch-morphologischen Bereich operiert. Für das Anspielen auf politische und wirtschaftliche Gegebenheiten ziehen die Autoren mehr oder weniger unpolitische und unökonomische Situationen heran und spielen auf sprachlicher Ebene mit polysemen oder (quasi-, partiell-) homophonen Eigenschaften von Wörtern oder mit Wortkreuzungen ${ }^{8}$. Anspielungsbasis in folgenden Beispielen sind die Nachnamen von Harry Tisch, Günter Mittag, Egon Krenz und Helmut Kohl, deren Namen bereits bestehende und semantisch besetzte Wörter aufgreifen (die Wortspieltypen sind hervorgehoben):

Beispiel 38:

Harry mußte vom Tisch (taz 01.11.1989, S. 8)

Basiswendung: vom Tisch müssen bzw. etwas vom Tisch wischen

Beispiel 39:

„Krenz zu Tisch!“ (taz 06.11.1989, S. 2)

Basiswendung: $z u$ Tisch bitten

Beispiel 40:

„Wer keinen Tisch mehr hat und keinen Mittag, kann auch aufs KaffeeKrenzchen verzichten " (taz 06.11.1989, S. 2)

Basiswendungen: zu Mittag essen, Kaffeekränzchen, Berliner Kränzchen, einen Tisch haben

$\mathrm{Zu}$ den formalen Aspekten von Wortkontaminationen vgl. ausführlich GoŁĘBIOWSKI (2011:210-221). 
Beispiel 41:

Berliner Krenzchen mit Mischnick. FDP-Fraktionschef überwältigt von Besuch bei Egon Krenz (taz 26.10.1989, S. 2)

Basiswendung: Berliner Kränzchen

Beispiel 42:

„Helmut, bau deinen Kohl im Westen an, weil man ihn hier nicht brauchen kann “ (taz 21.12.1989, S. 2)

Basiswendung: Blumenkohl anbauen

Die Wortspiele in folgenden Beispielen beruhen auf lautlicher Ähnlichkeit; der Name Kohl wird Bestandteil eines anderen Wortes:

Beispiel 43:

Niemand weh und allen wohl - ein deutscher Furz von Coca-Kohl!

(taz 08.12.1989, S. 21)

Beispiel 44:

„Unsere DDR - keine Kohlonie “ (taz 21.12.1989, S. 2)

Beispiel 45:

„Gegen Verkohlung “ (taz 21.12.1989, S. 2)

Nachfolgend beruhen die Wortspiele auf der Substitution oder Hinzufügung eines Lautes, die Anspielung bezieht sich auf Egon Krenz:

Beispiel 46:

Gegen den ,Ego(n)ismus “ (taz 25.10.1989, S. 15)

Beispiel 47:

Schluß mit der Ego(n)zentrik! (LANG 1990:82)

Beispiel 48:

„Ökonomie statt Egonomie “ (taz 25.10.1989, S. 3)

In drei weiteren Beispielen liegt Substitution eines Lautes vor. Es handelt sich bei den ersten beiden um Anspielungen auf den Namen Krenz, die ,grenzenlos“" und „unbegrenzt“" im Sinne von unendlich, uneingeschränkt abwandeln; im dritten Beispiel um ein Wortspiel mit dem Namen Sarkozy und dem Wort Zar:

Beispiel 49:

„Sozialismus krenzenlos “ (taz 25.10.1989, S. 3)

Beispiel 50:

Unbekrenzte Macht den Räten! (taz 06.11.1989, S. 2) 
Beispiel 51:

Zarkosy; Nicht nur mit seinem Reformwillen eifert Sarkozy einem historischen Vorbild nach. Napoleon lenkte und disziplinierte die Kultur - zu Sarkozys politischem Verständnis gehört die Kontrolle der Medien: der öffentlich-rechtlichen, die dem Staat - ergo: ihm selber - unterstehen, wie der privaten, die seinen Freunden gehören. „Zarkosy“ lautet der erste Kosename des neuen Präsidenten. (F.A.Z. 04.07.2007, S. 36)

Anspielung auf die Namen Kohl, Krenz und Brandt; Wortkreuzungen von Kohl + Suppe, Kohl + Plantage, Kohl + Waggon, Krenz + Grenze + Gebiet , Brandt + brandaktuell:

Beispiel 52:

Große Teile des Volkes glauben an das Wunder der Wiedervereinigung, das alle Probleme löst. Das führt unter anderem auch zu Massendemonstrationen für „, Großdeutschland”. In dieser Situation, wo für alle sichtbar die Zeitbombe tickt, kann diese Regelung der Zünder sein, der die Explosion auslöst. [...] Wir wollen nicht in die Kohlsuppe rein! (taz 14.12.1989, S. 20)

Beispiel 53:

Ansonsten rollt der Kohl-band-Waggon (taz 01.12.1989, S. 8)

Beispiel 54:

Der deutsche Kolonialismus erlebt eine neue Blütezeit: Deutsches Streben nach unerschlossenem Raum im Osten ist wieder in! Die Statthalter rüsten auf zu großen Antrittsbesuchen in ihren künftigen Provinzen - als humaner Akt von gnadenloser Güte. Die Vision einer großen Kohlplantage im gesamten Krenzgebiet beherrscht die Köpfe und Krägen. (taz 08.12.1989, S. 21)

Beispiel 55:

Die DDR ist keine Kohl-Plantage (taz 11.12.1989, S. 17)

Beispiel 56:

„Brandtaktuell für die DDR: gegen die Wiedervereinigung “ (taz, 21.12.1989, S. 9) Wortkreuzung von teuer / Teuerung + Euro:

Beispiel 57:

„,Teuro" als psychologischer Effekt. [...] Tatsächliche und gefühlte Teuerung im Euro-Raum (F.A.Z. 18.05.2007, S. 14)

Ein interessantes wortspielerisches Anspielungsverfahren stellt das Auflösen von offiziellen, allgemein bekannten Abkürzungen dar. ${ }^{9}$ Eine Variante ist das

$9 \quad$ Vgl. dazu BŁachut (2014:306-310). 
Akrostichon-Wortspiel, bei dem die Anfangsbuchstaben (Buchstaben bei Wortfolgen) so hintereinander ausbuchstabiert werden, dass sie gegenüber dem ursprünglichen Akronym einen neuen Sinn ergeben. Unter den dokumentierten Belegen sind zwei Typen zu verzeichnen. Zum einen können die neuen Lexeme (hervorgehoben) jeweils die gleiche Wortart wie die der ursprünglichen Vollform repräsentieren:

Beispiel 58:

DDR Deutsche Demonstrierende Republik (taz 01.11.1989, S. 7)

Beispiel 59:

Von der D(eutschen) D(emokratischen) R(epublik) in die B(unker) R(epublik) D(rängelland) (taz 16.11.1989, S. 3)

Beispiel 60:

SED - „S“ wie Sauwirtschaft, „E“ wie Egoismus und „D“ wie Diebstahl (taz 11.12.1989, S. 1)

Zum anderen entsteht ein neu strukturiertes Syntagma bzw. eine Wortgruppe, ein Satz oder Teilsatz (hervorgehoben), z. B.:

Beispiel 61:

BRD Bardei rädlischer Deutscher (taz 16.12.1989, S. 2)

Beispiel 62:

„,Müllgruppe BRD/DDR“ (Bloß Rüber Damit - Dreck Dankend Retour)

(taz 14.12.1989, S. 3)

Beispiel 63:

PDS - Partei Der Sauwirtschaft! (LANG 1990: 105)

Beispiel 64:

„SOS“ Sozialismus ohne Stasi (taz 16.12.1989, S. 2)

Beispiel 65:

Sie schlagen für die SED das Kürzel ,WSDP" vor Wir sind die Partei [...] oder statt SED jetzt DES Deutsche Einheitssozialisten (taz 16.12.1989, S. 2)

Beispiel 66:

„Trabi“" Tragische Arbeiter \& Bauern-Initiative (taz 16.12.1989, S. 2)

Ein anderes, viel selteneres Verfahren beruht darauf, dass eine gängige, ursprüngliche Kurzform in ein anderes Wort integriert wird, dazu zwei Beispiele:

Beispiel 67:

Wir lassen uns nicht BeeRDigen (taz 01.02.1989, S. 4) 
Beispiel 68:

Gegen die BRDigung der DDR (taz 09.12.1989, S. 29)

In allen Varianten manifestieren sich individuelle oder kollektive Stellungnahmen zu einem Objekt oder einem Sachverhalt, die aufgrund des verwendeten (neuen) Wortschatzes den Faktor einer expressiven und bewertenden Stellungnahme einschließen.

\subsection{Analogiebildungen}

Sprachliches Anspielen durch Analogie beruht auf dem Bilden oder Umbilden einer sprachlichen Form nach dem Muster einer anderen. Die Produkte der Analogiebildung können einmalig, okkasionell sein, wie die bereits zitierten Beispiele Gegen den „Ego(n)ismus“ (taz 25.10.1989, S. 15), Schluß mit der Ego(n)zentrik! (LANG 1990:82) oder ,Ökonomie statt Egonomie“ (taz 25.10.1989, S. 3), bei denen das Entstehen des Neuwortes durch die lautliche Ähnlichkeit motiviert ist. Die Forschung dokumentiert solche Neubildungen als Wortspiele. In diesem Unterkapitel werden Analogiebildungen im Bereich der Wortbildung und der Phrasenbildung diskutiert, die a) nicht wortspielerisch und nicht mehrdeutig im obigen Sinne, sondern b) im Gegenteil in hohem Maße regelhaft, dabei c) reihenbildend, aber trotzdem d) nicht weniger kreativ sind als Wortspiele. Der kommunikative Erfolg dieser Bildungen besteht darin, dass sie sich eines intuitiven, im Sprachbewusstsein verankerten Sprachwissens bedienen und sich leicht durchsetzen, denn sie scheinen kein Nachdenken zu erfordern. Sie komprimieren zugleich komplexe Sachverhalte.

Der Mechanismus für die Analogiebildung lässt sich so darstellen: A (Analogieprodukt) hat Ähnlichkeit mit B (Basis), B hat die Eigenschaft X. Also hat auch A die Eigenschaft X, vgl. die folgenden Reihen:

(Nach-) Name + -ismus/-ysmus

Die Nomina beziehen sich auf den Träger des Namens, aber durch ihre komprimierte Form auch auf seine Taten und Leistungen. Eine bestimmte Person wird als Autor einer philosophischen, ideologischen etc. Richtung, einer bestimmten, allgemein feststellbaren und anerkannten bzw. kritisierten Verhaltensweise hervorgehoben. Historisch gefestigt und mit Symbolwert versehen sind heute z. B. Leninismus, Marxismus, Stalinismus, Trotzkismus, Maoismus, Darwinismus, Kalvinismus etc. Analoge Bildungen sind: Sarkozysmus (F.A.Z. 18.05.2007, S. 2), Dreggerismus (taz 04.11.1989, S. 5), Thatcherismus (F.A.Z. 11.05.2007, S. 1; S. 8), Blairismus (F.A.Z. 30.04.2007, S. 2; 28.6.2007, S. 2), Baudrillardismus (taz 08.08.2007, S. 19), Gaullismus (taz 27.04.2007, S. 11), Spinozismus (taz 25.06.2007, S. 12) etc. 
$\mathrm{X}+$-phobie

Nach dem Vorbild einer bereits gefestigten, lexikalisierten Bildung (z. B. Homophobie, Xenophobie) entstehen kontextgebunden Neuwörter, die systemhaft die Bedeutung des Grundwortes übernehmen und durch die Wahl des Bestimmungswortes die kollektive oder individuelle Stellungnahme zu einem Objekt markieren. Analoge Bildungen sind: Es kam eine regelrechte „Schottophobie" unter den Engländern auf (F.A.Z. 30.04.2007, S. 44), Die EKD verstärkte die „Islamphobie“ in Deutschland (F.A.Z. 08.06.2007, S. 6).

$\mathrm{X}$ à la [(Nach-) Name]

Durch umgangssprachliche Formulierung mit à la (d. h. im Stile von, auf eine bestimmte Art), dem ein Eigennamen angekoppelt wird, werden die Sachverhalte im jeweiligen Situationskontext und je nach Sprecherperspektive (Befürworter / Gegner der aktuellen Politik, einer Entscheidung etc.) bzw. in Bezug auf die Symbolhaftigkeit der betreffenden Person charakterisiert und auch bewertet. Verwendungskontexte zeigen, dass diese komprimierte Art des Anspielens auf komplexe Sachverhalte häufiger dem Verspotten, Ironisieren, Bezweifeln, seltener dem Loben dienen. Analoge Reihenbildungen (meistens aus den Kopfzeilen oder zeittypische Losungen) sind: Dialog und Reformen à la Erich [Honecker] sind dem Volk zu spärlich (taz 18.10.1989, S. 1), Reformen à la Krenz (taz 10.11.1989, S. 3), Reformen a la [sic!] Hager sind uns zu mager (taz 18.10.1989, S. 2), Ellbogengesellschaft à la Thatcher (taz 27.11.1989, S. 5), die Einschüchterung à la Sarkozy (taz 19.04.2007, S. 18), die Experimente à la Dolly (taz 08.06.2007, S. 8), Wohlfahrtsstaat à la Bismarck (taz 24.05.2007, S. 11) etc.

\subsection{Anspielen ohne erkennbare Anspielungsbasis}

Am schwierigsten in der Rezeption sind Anspielungen, bei denen sich die Empfänger auf nichts Formelhaftes, auf keinen bekannten Ausdruck, auf keine Analogie stützen können. Hier sind ein entsprechendes Weltwissen und eine gewisse Sprachsensibilität erforderlich. Hinzu kommt, dass die Möglichkeit, dass die Anspielung als solche erkannt wird, umso größer ist, je inhaltlich einheitlicher der Diskurs ist (im Sinne von einer Reihe thematisch zusammenhängender Texte).

Die Formulierungen Visafrei bis Hawaii! (taz 06.11.1989, S. 2), Ohne Visa bis nach Pisa! (taz 06.11.1989, S. 2), Visafrei bis Shanghai! (LANG 1990:104) oder Freies Surfen auf der Ostsee! (LANG 1990:104) sind indirekt formulierte Forderungen, bei denen das ,Mehr' ihrer Aussagekraft nur bei Kenntnis der 
politisch-ökonomischen und - aus heutiger Perspektive - historischen Ereignisse abgeleitet werden kann. Reimbindung und Rhythmisierung sind stilistische Mittel, die alleine noch keine Garantie für das Erkennen einer Anspielung sind. Der Sinn der Anspielung liegt in der Einordnung von Hawaii, Pisa, Shanghai, Ostsee in diesem konkreten Situationskontext als Metonymie für ,eine Welt ohne Grenzen‘.

\section{Funktionen von Anspielungen}

Man kann es nicht adäquater formulieren als WILSS (1989:VII), der feststellte, dass in Anspielungen „Wirklichkeit dargestellt, verfremdet, kritisiert, ironisiert, glossiert oder absichtsvoll verändert wird“. Man kann allerdings seine Bemerkung hauptsächlich auf Topoi als Anspielungsbasis auf alle, formal wie inhaltlich differente Anspielungstypen und Anspielungsverfahren übertragen, denn diese Spannung zwischen Gesagtem (als Anspielung) und wirklich Gemeintem ist immer durch die Auseinandersetzung mit der umgebenden Wirklichkeit motiviert. Die Gründe für den Gebrauch von Anspielungen sind verschieden. Ebenso verschieden sind auch die Funktionen von Anspielungen, die Empfänger ihrer Auffassungsgabe gemäß diesen Anspielungen zuordnen wollen. Im Folgenden werden die erkennbaren Funktionen der zitierten Anspielungstypen diskutiert.

\subsection{Kritisieren, Ironisieren, negatives Bewerten}

Die zitierten Belege gruppieren sich thematisch um politische und wirtschaftliche Kontexte. Dabei wird in oder durch Anspielungen jeweils so etwas wie ein Zeitgeist widergespiegelt, der in den dokumentierten Beispielen am häufigsten als Kritik, Ironie oder die negative Bewertung eines Sachverhalts oder einer für etwas zuständigen Person ausfällt. Was dabei häufig mitschwingt, ist die mehr oder weniger direkte Forderung nach Veränderung.

Beispiel 69: metaphorisches Bild aus dem Konzept Schachspiel

Defensiver Schachzug [Überschrift] [...] Die Übernahme der Borsa Italiana ist nichts anderes. Es ist ein defensiver Schachzug. Er macht es der amerikanischen Nasdaq, die sich gegen den Willen von Furse mit 30 Prozent an der LSE beteiligt hat, wesentlich schwerer, den Rest der LSE zu kaufen. Furse hat damit zunächst die Unabhängigkeit der Londoner Börse gerettet. Kein Wunder, dass die Londoner Aktionäre dem sofort zustimmen. (F.A.Z. 25.06.2007, S. 11) 
Das Bild des Schachzugs wird im dadurch komprimierten Sinn von ,einer (hier: defensiven, Bewertungsadjektiv) Handlung zur Erreichung eines bestimmten und dem eigenen Interesse dienenden Ziels ${ }^{6}$ zur Demaskierung eines Geschehens verwendet, das der Öffentlichkeit als , großer Schritt der Konsolidierung' dargestellt und als solcher auch öffentlich gefeiert wurde. Die Wahl der Metapher und auch die hinzukommenden kommentierenden Wörter und Ausdrücke (defensiv, $\mathrm{X}$ ist nichts anderes, kein Wunder etc.) geben die individuelle, kritische Sichtweise des Autors wieder.

Beispiel 70: Anspielung durch Analogiebildung (mit Reimbindung)

Dem Land ein neues Antlitz - ohne Kalk aus Wandlitz (taz 06.11.1989, S. 2)

Kommt raus aus Wandlitz, seht uns ins Antlitz. (taz 18.11.1989, S. 25)

Der Ort Wandlitz bei Berlin als Anspielungsobjekt verdankt seine deutschlandweite Bekanntheit den prominenten Mitgliedern des Politbüros der SED, die in der nahe gelegenen Waldsiedlung wohnten. Der Ort wird zum Gegenstand von Ironie und Witz, wobei auf indirekte Weise auf ein konkretes politisches Geschehen angespielt wird: auf die politische Kursänderung nach Honeckers Rücktritt. Die Losungen enthalten negative Gefühle gegenüber den Bewohnern von Wandlitz. Sie sind als Forderungen formuliert (Illokutionsindikatoren der Forderung sind hier: Verben im Imperativ, Dativ zur Markierung der Aufforderung, Bedingungen markiert durch ohne $\mathrm{X}$, ein neues $\mathrm{X}$ ).

\subsection{Warnen}

Es lassen sich auch Warnungen nachweisen, dazu ein Beispiel:

Beispiel 71: Anspielung auf ein Zitat

Mit Mühe bringen verschüchterte Funktionäre Flugblätter - ,Was wir wollen“ unters Volk. Merkzettelchen - ,Wollt ihr den totalen Kohl “- werden den Verteilern aus der Hand gerissen und vor ihren Augen zertrampelt. [...] (taz 23.12.1989, S. 37) Mit dem Satz „Wollt ihr den totalen Kohl“ (unverkennbar als Warnung intendiert) wird möglicherweise auf den Ausdruck der totale Staat (nach dem Staatsrechtler Carl Schmitt 1932 in seiner Schrift Der Begriff des Politischen) angespielt, der für ein totalitäres und antiliberales Staatsmodell steht. ${ }^{10}$ Der neue Ausdruck soll vor der Person warnen, deren Entscheidungen und Regelungen befürchtet werden. Mit der Frage appelliert der Autor an das von ihm

10 Und formal wohl auch auf Goebbels Sportpalastrede Wollt ihr den totalen Krieg?! von 1943. Hier nach: Joseph Goebbels, Rede im Berliner Sportpalast [Wollt Ihr den totalen Krieg], 18.02.1943, http://www.1000dokumente.de/index.html/index.html?c =dokument_de\&dokument=0200_goe\&object=translation\&l=de (13.05.2017). 
und seinen Adressaten geteilte Wissen bezüglich der für sie selbstverständlichen negativen Antwort, hinter der zugleich ein konkretes Bewertungsresultat steht.

\subsection{Aufmerksamkeit wecken}

Es muss festgehalten werden, dass nicht alle spielerisch formulierten Anspielungen vordergründig der Kritik, der Ironie oder der Realisierung bestimmter Bewertungsstrategien dienen. Oft sind sie einfach Resultat der Freude des Autors am Wortspiel und des Spiels mit der Sprache. Mit schmückender und Aufmerksamkeit heischender Funktion werden sie besonders häufig in Pressetexten gebraucht, bei denen die Anordnung der Textelemente einer Planung unterliegt, so z. B. in Zeitungsüberschriften, wo sie durch ihre Form zum Weiterlesen anregen sollen, oder am Ende des Textes, wo sie eine interpretierendzusammenfassende Funktion haben.

Beispiel 72: metaphorisches Bild aus dem Konzept Tanz

Rumba zu einer neuen Mitte [Überschrift] Nun hat Brown einen Schritt nach rechts getan und den Liberalen eine Koalition angeboten, doch die haben das abgelehnt. Dann machte er einen Schritt nach links und versprach eine sozialere Wohnungs-, Gesundheits- und Ausbildungspolitik. Er tat einen Schritt nach vorn und stellte ein aktiveres Management für Klimaschutz und Wettbewerbsanpassung in Aussicht. Und dann tat er einen Schritt „,hinunter" und versprach einen effektiven Dialog mit der Gesellschaft [...] Sofort sprach er aber auch davon, dass er den Einfluss der Gewerkschaften auf die Labour-Partei weiter verringern wolle [...] Wohin und wie weit Browns Rumba-Tanz führen wird, bleibt abzuwarten, eines ist aber sicher: Wenn er gelingt, dann führt er zu einer neuen Mitte. (F.A.Z. 28.06.2007, S. 2)

Mit der Wahl der Metapher als Beschreibung für außersprachliche Sachverhalte und Zusammenhänge wird die Aufmerksamkeit der Rezipienten auf besondere, mit Absicht betonte Aspekte der Wirklichkeit gelenkt, die der Autor für wichtig hält. Mit allgemein bekannten und gängigen Ausdrücken als Anspielungsinstrument wird ein Vertrauensfeld aufgebaut, es wird suggeriert, dass Ereignisse in der Politik oder in der Wirtschaft nicht so kompliziert seien, wie sie auf den ersten Blick erscheinen mögen.

\subsection{Manipulieren, Beeinflussen der Meinung}

Für die Erklärung dieser Funktion kann man annehmen, dass der Autor, wenn er auf ein Bild aus dem Alltag, auf eine bekannte, allgemeingültige und Wahrheit beanspruchende Wendung anspielt (und sie dazu noch modifiziert), dies 
nicht nur aus Spaß und Freude am Sprachspiel selbst macht, sondern zugleich auf den Rezipienten einwirken und ihn in eine bestimmte Richtung lenken will. Außer ästhetisch anzusprechen, kann damit auch relativiert, getäuscht, irregeführt, distanziert, nuanciert, etwas in Frage gestellt, verfälscht, emotional angesprochen und entsprechend beeinflusst werden etc. Alltägliche, einfache Bilder und Wendungen mit einprägsamer, modellhafter Form sind dazu sehr gut geeignet, weil sie als Schablonen dienen, mit denen willkürliche Inhalte und Bewertungen transportiert werden können, die zuallererst seinen eigenen Interessen / Einstellungen entsprechen. Häufig fokussiert die eigentliche dichterische und stilistische Funktion der umgestalteten (erkannten) Formulierung die Aufmerksamkeit der Rezipienten in erster Linie auf die Form des Ausdrucks, schwächt ihre intellektuellen Reaktionen auf den Inhalt ab und schränkt damit ihre Kritikfähigkeit ein.

KOLLER (1975) hat in Bezug auf Redensarten die These aufgestellt, dass sie wegen ihrer Kürze und Prägnanz ,generell die Funktion der zusammenfassend-interpretierend-wertenden Vorwegnahme des Hauptinhalts eines Beitrags [haben]; sie steuern damit die Rezeption des Beitrags selbst" (KOLLER 1975:404). Er nahm an, dass Redensarten enthaltende Schlagzeilen eine Stellungnahme des Schreibers enthalten und als Interpretationsanleitung und auch als Wertungsvorausnahme vor dem Hintergrund implizierter Interaktionsregeln dienen (können), indem sie dem Leser seine eigene Interpretation und seine eigene Bewertung der Fakten abnehmen (vgl. KOLLER 1975:405f.).

Beispiel 73: Anspielung auf eine Redensart

Mit Sparmaßnahmen ins Fettnapf getreten (Überschrift, Beispiel aus KOLLER 1975:403)

Die Redensart ins Fettnäpfchen treten drückt ein Verhaltensmuster aus dem Erfahrungsbereich des Sprechers aus. Durch die Übertragung auf einen politisch-ökonomischen Situationszusammenhang wird dieser auch implizit bewertet. Die Anspielung präsupponiert das Folgende: man sollte vermeiden, ins Fettnäpfchen zu treten.

In einer gewissen Erweiterung der Beobachtungen Kollers zitiere ich einen Textsauschnitt mit einer Redensart am Ende eines Textes; sie fordert einerseits den Leser zur Reflexion heraus, bewertet aber andererseits, und besonders an dieser Stelle, ohne die Möglichkeit, eine Eigenbewertung zuzulassen.

Beispiel 74: Anspielung durch die sprichwörtliche Redensart

Hinzu kommt nämlich, dass sich Energiesparlampen nur bei Dauerbetrieb lohnen, sodass man, wenn man die Lebensdauer der Lampe verlängern will, sie 
am besten den ganzen Abend brennen lässt - womit der Energiespareffekt auch zum Teil wieder aufgehoben ist. Man treibt den Teufel mit dem Beelzebub aus zugunsten einer wachsenden Industrie! (taz 16./17.06.2007, S. 11)

\subsection{Positives Bewerten}

Verschiedene Anspielungsverfahren werden auch dazu verwendet, Sachverhalte der umgebenden Welt positiv zu bewerten. Unten werden die Prädikativstrukturen zitiert, die am häufigsten für den metaphorischen Vergleich in Bezug auf Personen oder Sachen verwendet werden. Die Position der Anspielung ist hier ebenfalls wichtig. In der Schlagzeile / Überschrift und im Schlusssatz steuern die Anspielungen die Rezeption des Artikels, und dies auch im Hinblick auf die Bewertung des Thematisierten.

Beispiel 75: Prädikativstruktur in der Überschrift

[Foto der Übersetzerin, ihr Name: Sezer Duru, Überschrift:] Anwältin der deutschen Literatur (F.A.Z. 22.06.2007, S. 40)

Anlass für den Artikel war die Auszeichnung der Übersetzerin deutschsprachiger Schriftsteller ins Türkische mit dem DekaBank-Preis des Frankfurter Literaturhauses. Der Text selbst entfaltet die Schlagzeile in gleicher - positiver - Bewertungsrichtung. Eine emotionale Reaktion / positive Bewertung werden wohl alle Aussagen evozieren, die als Vergleichskern Berufsnamen oder Berufsfunktionen mit hohem gesellschaftlichem Prestige verwenden wie Anwalt oder (unten) Architekt.

Beispiel 76: Prädikativstruktur im Schlusssatz

Solana habe die „Methode der vernetzten Sicherheit" so perfektioniert, dass Europa als glaubwürdiger und berechenbarer Partner bei den Bemühungen um friedliche Konfliktlösungen erscheine. Solana sei ein ,Architekt für Frieden, für

Stabilität und für Demokratie“". (F.A.Z. 18.05.2007, S. 5)

Der Anlass für diesen Artikel war die Verleihung des Internationalen Karlspreises an den Beauftragten für die Außen- und Sicherheitspolitik der EU, Javier Solana. Auch hier wird im Fließtext ganz im Sinne des metaphorischen Vergleichs argumentiert.

\subsection{Textstrukturierende Funktion von Anspielungen}

Abschließend wird eine auffallende Funktion von Anspielungen besprochen, die sich in ihrer absichtlichen Positionierung im Text realisiert. Anspielungen werden in drei sequenzstrukturellen Positionen verwendet: in der Überschrift oder im ersten Satz des Textes bzw. des Abschnittes (als Ankündigungen, Einleitungen), im Fließtext (als Themenentfaltung, Kommentare) oder im 
Schlusssatz des Textes (als Konklusion, Zusammenfassung). Oft werden zwei Positionen, der erste und der letzte Satz eines Textes, mit derselben Anspielung besetzt. Ein Autor, der bekannte, formelhafte oder metaphorische Ausdrücke gebraucht, um z. B. politische oder wirtschaftliche Zusammenhänge zu erfassen und zu kommentieren, biedert sich gleichsam bei den Rezipienten in ihrer unmittelbaren Lebenswelt an und versucht sie für eine bestimmte Einstellung oder ein bestimmtes Verhalten zu gewinnen. Prinzipiell ist davon auszugehen, dass der Autor immer zweckorientiert verfährt, wenn er seine Reden oder schriftlichen Ausführungen mit Anspielungen einleitet, sie als Unterstützung seiner Argumente gebraucht oder diese mit ihnen zusammenfasst.

Beispiel 77: Anspielung durch ein metaphorisches Bild in der Überschrift (Ankündigung des Themas) und im Schlusssatz (Pointe)

Der Sündenbock [Überschrift] Tatsächlich ist es so, dass es vom Standpunkt Recht und Gerechtigkeit aus ziemlich egal ist, ob und wie Libby bestraft wird [...] [bezogen auf die Begnadigung des wegen Meineids verurteilten Lewis Libby durch Präsident Bush] Schließlich musste er allein den Kopf hinhalten für die vom Kollektiv um den Präsidenten und Vizepräsidenten zu verantwortende Abstrafung eines „Dissidenten“ “...] Die Massenvernichtungswaffenlüge selbst ist bereits in den Schubladen der Geschichte abgelegt. Zumindest einstweilen. Es wird später noch diverse Abrechnungen geben, juristisch relevant werden sie nicht mehr sein. Insofern kann man auf die Bestrafung eines einzelnen Sündenbocks wirklich verzichten. (F.A.Z. 05.07.2007, S. 2)

Beispiel 78: Anspielung durch ein metaphorisches Bild in der Überschrift (Ankündigung) und Entfaltung des Themas im Fließtext (Kommentar, Begründung)

Goliath Porsche [Überschrift] Die Hauptversammlung von Porsche hatte so gar nichts Feierliches. Sich komplizierte Rechtsgeschäfte beschreiben zu lassen ist nicht gerade spaßig, daher ist es nicht verwunderlich, dass sich die Reihen der Porsche-Vorzugsaktionäre früh lichteten, als über die Neugliederung des Konzerns diskutiert wurde. Doch das Datum wird in die Geschichte des Stuttgarter Unternehmens als Zäsur eingehen, als das Ende der David-Ära: Porsche ist jetzt endgültig nicht mehr der kleine, freche David, als den Porsche-Chef Wiedeking sein Unternehmen so gern charakterisiert hat. Der Sportwagenbauer ist seiner Nische entwachsen, ist Hauptaktionär bei Volkswagen geworden, einem wahren Goliath, wenn man in Wiedekings Bilderwelt bleibt. Da ist der Wunsch nachvollziehbar, die Strukturen so zu ordnen, dass bei dem renditestarken Sportwagenbauer nicht plötzlich sachfremde Interessen dominieren. (F.A.Z. 27.06.2007, S. 20) 
Beispiel 79: Anspielung durch die Redensart im ersten Satz (Einleitung zum Thema)

Wie der Teufel das Weihwasser scheuen die Taktiker in CDU und CSU das Etikett „,neoliberal“. Dass zwischen den Vorstellungen der Union und liberalen, marktwirtschaftlichen Rezepten tatsächlich Welten liegen, zeigte sich kürzlich in Potsdam bei einer Podiumsdiskussion der Hayek-Gesellschaft zur Gesundheitspolitik. (F.A.Z. 15.06.2007, S. 14)

\section{Ein kurzes Fazit}

In Zeitungsartikeln kommen Anspielungen (als Produkte des Anspielens) verschiedener Art sehr häufig vor. Autoren bedienen sich solcher Ausdrücke, bei denen Konnotationen aus Erfahrungen des Alltags und aus literarischem oder geschichtlichem Hintergrundwissen hervorgehen. Ob in ihrer ursprünglichen oder in der modifizierten Form, Anspielungen sind (als Instrumente und als Produkte des Anspielens) allesamt Ausdrücke, durch die sich die Einstellung des Sprechers zum beschriebenen Sachverhalt manifestiert. Die Rezeption von Anspielungen hängt immer von der sprachlichen Kompetenz der Sprecher ab. Sprachsensibilität ist eine Voraussetzung, die eine Kombination ist aus der Anwendung von Sprach- und Weltwissen einerseits (Rezipienten unterscheiden sich in ihren kognitiven Eigenschaften, sie können ein unterschiedliches Geschichtsbewusstsein haben etc.) und Annahmen über das, was der Verfasser mit der Wahl der Ausdrucksform vermitteln wollte andererseits. In kommunikativ-pragmatischer Hinsicht können Anspielungen dem Leser die eigene Interpretation und die eigene Bewertung der Fakten abnehmen, und zwar durch Implikaturen und Präsuppositionen (als pragmatische Prinzipien der Kommunikation) und durch spezifische Positionierung der Anspielungen (auf der Textebene).

\section{Literatur}

BŁACHUT, EDYTA (2004): Sprachspielerische Modifikationen formelhafter Wendungen. Untersuchungen anhand deutscher und polnischer Belege. Wrocław.

- (2008): Wertende Konnotationen von Metaphern. Am Beispiel von Metaphern aus politischen Kommentaren zur Wiedervereinigung Deutschlands 1989. In: Studia Linguistica XXVI: 27-48.

- (2014): Bewerten - Semantische und pragmatische Aspekte einer Sprachhandlung. Hamburg. 
Burger, Harald (1998/2003): Phraseologie: eine Einführung am Beispiel des Deutschen. Berlin (= Grundlagen der Germanistik 36).

Duden. Deutsches Universalwörterbuch ( $\left.{ }^{8} 2015\right)$. Mannheim / Leipzig et alii.

Fleischer, WolfGANG (1997): Phraseologie der deutschen Gegenwartssprache. 2., durchgesehene und ergänzte Auflage. Tübingen.

FRIELING, GUDRUN (1996): Untersuchungen zur Theorie der Metapher. Das Metaphern-Verstehen als sprachlich-kognitiver Verarbeitungsprozeß. Osnabrück.

GolęBIOWSKI, ADAM (2011): Kontamination: Form - Semantik - Pragmatik. Dresden / Wrocław.

KOLLER, WERNER (1975): Redensarten in Schlagzeilen. In: Muttersprache 85: 400-408. LANG, EwAlD (1990): Wendehals und Stasi-Laus. Demo-Sprüche aus der DDR. München.

PoHL, INGE (2002): Kognitive Metapherntheorie inklusive Frameansatz als Beschreibungsinstrumente metaphorischer Projektion, dargestellt an Metaphern aus meinungsbildenden Texten. In: POHL, INGE (ed.): Semantische Aspekte öffentlicher Kommunikation. Frankfurt a. M. / Berlin et alii, 105-143.

PTASHNYK, STEFANIYA (2005): ,Unstabile“ feste Wortverbindungen: Zur Dynamik des phraseologischen Sprachbestandes. In: Hermes, Journal of Linguistics 35: 77-95.

- (2009): Phraseologische Modifikationen und ihre Funktionen im Text. Eine Studie am Beispiel der deutschsprachigen Presse. Essen (= Phraseologie und Parömiologie 24).

RÖMER, RuTH (1977): Die Anspielung als Sprechakt. In: Muttersprache 87/6: 396-412.

SAUER, NiCOLE (1998): Werbung - wenn Worte wirken. Ein Konzept der Perlokution, entwickelt an Werbeanzeigen. Münster / New York et alii (= Internationale Hochschulschriften 274).

VATER, HEINZ (2010): Sprachspiele: kreativer Umgang mit Sprache. In: Linguistische Berichte 221: 3-36.

WILSS, WOLFRAM (1980): Beobachtungen zur Anspielungstechnik in der deutschen Gegenwartssprache. In: Grazer Linguistische Studien 11/12. Festgabe für Norman Denison: 368-390.

- (1989): Anspielungen. Zur Manifestation von Kreativität und Routine in der Sprachverwendung. Tübingen. 